\title{
Contenido de compuestos antioxidantes en tres estados de maduración de tomate de árbol (Solanum betaceum Cav.) cultivado a diferentes alturas (m.s.n.m.)
}

\section{(Antioxidant compounds content in red-purple tree tomato grown at different altitudes (m.a.s.I.))}

\author{
Lorena Cuesta ${ }^{1}$, María José Andrade ${ }^{1}$, Carlota Moreno ${ }^{1}$, Analía Concellón ${ }^{2}$
}

\begin{abstract}
Resumen:
El objetivo de esta investigación fue estudiar el contenido de compuestos antioxidantes en tomate de árbol morado cultivado a diferentes alturas (m.s.n.m.). Se emplearon frutos provenientes de Pelileo (2660 m.s.n.m.) y Chiquicha (2440 m.s.n.m.) en tres estados de maduración (inmaduros, maduros y sobremaduros). Se realizaron análisis fisicoquímicos (color superficial e interno, $\mathrm{pH}$, acidez total titulable -ATT- y sólidos solubles totales -SST-) y bioquímicos por espectrofotometría UV-Vis (fenoles totales -FT-, antocianinas totales -AT-, carotenos totales -CT-, ácido L-ascórbico -AA- y capacidad antioxidante -CA-) en el endocarpio y mesocarpio de los frutos. En el color se obtuvieron mayores valores de $L^{*}$ (epicarpio) y $h^{*}$ (epicarpio y mesocarpio) en frutos inmaduros, maduros y sobremaduros de Chiquicha; mientras que el valor de $\mathrm{C}^{*}$ del mesocarpio y endocarpio fue mayor en aquellos de Pelileo. Las diferencias de color fueron evidentes con la maduración, pero no se encontró una relación directa entre la altura de cultivo y los cambios en los parámetros de color analizados. Se obtuvo una diferencia significativamente mayor en el $\mathrm{pH}$ de tomates (inmaduros y maduros) de Chiquicha. Asimismo, estos frutos presentaron mayor ATT (maduros y sobremaduros) y SST (sobremaduros) que los de Pelileo. El mesocarpio presentó mayor contenido de FT y CT en los frutos (tres estados de maduración) de Chiquicha, mientras que mayor concentración de estos compuestos presentó el endocarpio de frutos sobremaduros de Pelileo. El contenido de AT y AA fue mayor en frutos maduros de Chiquicha. En general, los frutos de Chiquicha mostraron mayor concentración de compuestos antioxidantes; posiblemente porque dicha plantación, constituida por al menos dos genotipos, se sitúa en una zona montañosa con buenas características para el cultivo de este frutal.
\end{abstract}

Palabras clave: Tomate de árbol morado, altura de cultivo, maduración, colorimetría, compuestos antioxidantes.

\begin{abstract}
:
The aim of this research was to study the antioxidant compounds content in Red-purple tree tomato grown at different altitudes (m.a.s.l.). Fruits from Pelileo (2660 m.a.s.l.) and Chiquicha (2440 m.a.s.l.) in three maturity stages (unripe, ripe and overripe) were used. Physicochemical (surface and internal color, pH, titratable total acidity -TTA- and total soluble solids -SST-) and biochemical analyses using UV-Vis spectrophotometry (total phenol -TF-, total anthocyanins TA-, total carotenes -CT-, L-ascorbic acid -AA- and antioxidant capacity -AC-) in the mesocarp and endocarp of fruits were performed. In the color, higher values of $L^{*}$ (epicarp) and $h^{*}$ (epicarp and mesocarp) in unripe, ripe and overripe fruits from Chiquicha were obtained, while $\mathrm{C}^{*}$ values of mesocarp and endocarp was higher in those from Pelileo. The color differences were evident during fruit ripening, but a direct relationship between crop altitude and changes in
\end{abstract}

\footnotetext{
${ }^{1}$ Universidad Tecnológica Equinoccial, Facultad de Ciencias de la Ingeniería, Quito - Ecuador (acmj2221@ute.edu.ec)

${ }^{2}$ Universidad Nacional de La Plata, Centro de Investigación y Desarrollo en Criotecnología de Alimentos, La Plata - Argentina (aconcell@quimica.unlp.edu.ar)
} 
color parameters analyzed wasn't found. There was a significantly higher difference in the $\mathrm{pH}$ of tomatoes (unripe and ripe) from Chiquicha. Also, these fruits had higher TTA (ripe and overripe) and TSS (overripe) than those from Pelileo. The mesocarp showed higher content of TF and TC in the fruits (three maturity stages) from Chiquicha, while higher concentration of these compounds showed the endocarp from Pelileo's fruit. The TA and AA content was higher in ripe fruits from Chiquicha. In general, the fruits from Chiquicha showed higher concentration of antioxidant compounds, possibly because the plantation, consisting of at least two genotypes, is located in a mountainous area with good characteristics for this fruit cultivation.

Keywords: Red-purple tree tomato, crop height, maturity, colorimetry, antioxidant compounds.

\section{Introducción}

El tomate de árbol (Solanum betaceum Cav.) es un arbusto nativo de la región Andina de Sudamérica que se cultiva por su fruto tropical semiácido, considerado exótico debido a su agradable aroma y sabor agridulce. En Ecuador, crece en altitudes óptimas comprendidas entre 1500 y 2600 m.s.n.m. (Revelo, Pérez, \& Maila, 2004). Tungurahua es la provincia de mayor producción con 8300 ha (Ramírez, 2009), donde predominan los genotipos Amarillo gigante y el híbrido Mora ecuatoriano (Revelo et al., 2004), los mismos que se muestran en la Figura 1. Sin embargo, los cultivares constituidos por al menos dos genotipos han causado una hibridación y mezcla del material genético, reflejados en la heterogeneidad en las formas, tamaños y colores de los frutos (Chalampuente \& Prado, 2005). El color del tomate de árbol depende de compuestos con conocida actividad antioxidante como: carotenoides, flavonoides y antocianinas (Reyes \& Sanabria, 1993).
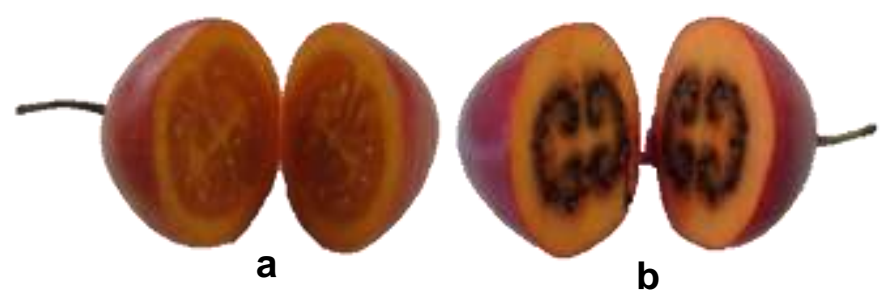

Figura 1. Tomate de árbol genotipos a) Amarillo y b) Mora

No se reportan estudios que relacionen el efecto de la ubicación altitudinal del cultivo sobre el contenido de compuestos antioxidantes durante la maduración de tomate de árbol. Pero se conoce que la maduración de los frutos, en general, está influida por el genotipo, edad del árbol, suelo, clima y prácticas culturales (Coleto, 1994); asimismo la maduración puede ejercer un efecto sobre la cantidad de compuestos antioxidantes y el color que los frutos puedan contener (Lee \& Kader, 2000; Prohens, Ruiz, \& Nuez, 1996; Rodríguez, 1999). También se sabe que los árboles ubicados a elevadas alturas han desarrollado un sistema antioxidante que los protege contra el estrés ambiental (irradiación alta, clima extremo, incremento de concentraciones de ozono, oxidantes atmosféricos, entre otros) (Hecke et al., 2003). En relación a este aspecto, se han realizado estudios en materiales vegetales como: aceite de oliva chétoui (Ben et al., 2012), uvilla (Fischer, Ebert, \& Lüdders, 2000), papas (Hamouz, Lachman, Dvorak, Cepl, \& Sarec, 2006) y Aconogonum weyrichii (Sakata, Nakato, \& Yokoi, 2006). Por lo que, el principal propósito del 
presente trabajo es identificar si el proceso de estrés altitudinal permite obtener frutos con mayor concentración de compuestos bioactivos, cuyo consumo se ha asociado con la prevención de enfermedades degenerativas, cardiovasculares y cancerígenas.

\section{Materiales y métodos}

Material vegetal: Tomates de árbol (Solanum betaceum Cav.) genotipo Morado cosechados en Pelileo (2660 m.s.n.m.) y Chiquicha (2440 m.s.n.m.), provincia de Tungurahua-Ecuador se trasladaron al Laboratorio de Química de Alimentos de la Universidad Tecnológica Equinoccial, donde se dividieron en tres grupos: inmaduros (75\% de color superficial rojo-púrpura), maduros (100\% de color superficial rojo-púrpura) y sobremaduros (almacenados 17 días a temperatura ambiente). En cada grupo se realizaron análisis fisicoquímicos (color superficial e interno, $\mathrm{pH}$, acidez total titulable -ATT- y sólidos solubles totales -SST-) y bioquímicos (fenoles totales -FT-, antocianinas totales -AT-, carotenos totales -CT-, ácido L-ascórbico -AA- y capacidad antioxidante total -CA-). Los análisis bioquímicos se efectuaron en el mesocarpio y endocarpio de los frutos.

Color: Se registró el color del epicarpio, mesocarpio y endocarpio de cada fruto con un colorímetro triestímulo (Konica Minolta Chroma Meter CR-400), para calcular los valores de la escala CIE $L^{*} C^{*} h *$. Los parámetros de color se obtuvieron empleando las siguientes ecuaciones (Sanz \& Gallego, 2001).

$$
\begin{aligned}
& L^{*}=116\left(Y / Y_{n}\right)^{1 / 3}-16 \\
& C^{*}=\left(a^{* 2}+b^{* 2}\right)^{1 / 2} \\
& h^{*}=\operatorname{arctg}\left(b^{*} / a^{*}\right)
\end{aligned}
$$

pH: Porciones de frutos se homogenizaron en un minipimer Philips para obtener un jugo que se filtró a través de una gasa de algodón. Se mezclaron $5 \mathrm{~g}$ del filtrado con $50 \mathrm{~mL}$ de agua destilada. $\mathrm{El}$ pH se determinó con un potenciómetro (Thermo Scientific: Orion Star) por inmersión del electrodo en la muestra.

Acidez total titulable: La muestra de $5 \mathrm{~g}$ de jugo de tomate de árbol y $50 \mathrm{~mL}$ de agua destilada, se tituló con $\mathrm{NaOH} 0.1 \mathrm{~N}$ hasta el viraje del pH a 8.2 empleando un potenciómetro (Thermo Scientific: Orion Star). La cuantificación de ATT se determinó según la ecuación 4 (Paltrinieri, Figuerola, \& Rojas, 1993).

$$
A=(V \times N \times 1000) / m
$$

Donde:

A: acidez, expresada como $\mathrm{meq} / \mathrm{kg}$

$\mathrm{V}$ : volumen de $\mathrm{NaOH}$ gastado 
$\mathrm{N}$ : normalidad de la solución de $\mathrm{NaOH}$

m: peso (g) de la muestra.

Sólidos solubles totales: Se colocaron entre dos y tres gotas de jugo de tomate de árbol en un refractómetro $\mathrm{B} \& \mathrm{C}\left(0-32^{\circ} \mathrm{Brix}\right)$.

Fenoles totales: $2.0 \mathrm{~g}$ de mesocarpio o $0.5 \mathrm{~g}$ de endocarpio triturado congelado se homogenizó con $10 \mathrm{~mL}$ de etanol en un agitador magnético durante 20 minutos. La mezcla se introdujo en una centrífuga (Hermle Labortechnik) a $6000 \mathrm{rpm}$ durante 20 minutos. La extracción se realizó a $4^{\circ} \mathrm{C}$, protegiéndose de la luz. El extracto se almacenó a $-20^{\circ} \mathrm{C}$ hasta su posterior análisis. La determinación de FT se realizó según el método de Singleton \& Rossi (1965) con ligeras modificaciones. Una alícuota del extracto (80 $\mu \mathrm{L}$ de mesocarpio o $90 \mu \mathrm{L}$ de endocarpio) se transfirió a un tubo de ensayo que contenía $1120 \mu \mathrm{L}$ o $1110 \mu \mathrm{L}$ de agua bidestilada, respectivamente. Se añadieron $100 \mu \mathrm{L}$ de la solución de Folin-Ciocalteu y agua destilada (1:1), se homogenizó en un vortex, y se mantuvo a temperatura ambiente por $3 \mathrm{~min}$. El contenido se mezcló con $200 \mu \mathrm{L}$ de $\mathrm{Na}_{2} \mathrm{CO}_{3} 20 \%$ p/v en NaOH $0.1 \mathrm{~N}$, se cubrió con papel film y permaneció a temperatura ambiente durante $60 \mathrm{~min}$ antes de medir su absorbancia a $760 \mathrm{~nm}$ en un espectrofotómetro (Génesis 20-Thermo Spectronic). Cada extracto se analizó por triplicado. La cuantificación se realizó con base a una curva patrón de ácido gálico.

Antocianinas totales: $0.20 \mathrm{~g}$ de mesocarpio o endocarpio triturado congelado se mezclaron con $10 \mathrm{~mL}$ de metanol- $\mathrm{HCl} 1 \%$ durante $10 \mathrm{~min}$ a $4^{\circ} \mathrm{C}$, y posteriormente se introdujo en una centrífuga (Hermle Labortechnik) a $6000 \mathrm{rpm}$ durante $10 \mathrm{~min}$ a $10^{\circ} \mathrm{C}$. El sobrenadante se filtró con papel filtro y del pellet se realizó otra extracción bajo los mismos parámetros. El extracto se aforó con el solvente a $30 \mathrm{~mL}$. La extracción se efectuó protegiéndose de la luz. La determinación de AT se realizó mediante un barrido espectral en un espectrofotómetro (Thermo Scientific Evolution 60S), previo a la lectura de su absorbancia a $530 \mathrm{~nm}$ (espectrofotómetro Génesis 20-Thermo Spectronic). La cuantificación de AT se determinó según Beas et al. (2011), utilizando la ecuación 5 .

$$
C=\left(\frac{A}{E}\right)\left(\frac{V o l}{1000}\right)(P M)\left(\frac{1}{\text { peso de la muestra }}\right)\left(10^{6}\right)
$$

Donde:

C: concentración de antocianinas totales $(\mathrm{mg} / \mathrm{g})$

A: absorbancia máxima

$\varepsilon$ : absortividad molar de la cianidina 3-glucósido $\left(25965 \mathrm{~cm}^{-1} \mathrm{M}^{-1}\right)$

Vol: volumen total del extracto de antocianinas

PM: peso molecular de cianidina 3-glucósido (449 Da). 
Carotenos totales: La extracción y cuantificación de CT se realizó según Arango (2006). Aproximadamente $1.0 \mathrm{~g}$ de mesocarpio o $0.35 \mathrm{~g}$ de endocarpio triturado congelado se mezcló con $2.5 \mathrm{~mL}$ de éter de petróleo y $2.5 \mathrm{~mL}$ de acetona durante $20 \mathrm{~min}$ a $4^{\circ} \mathrm{C}$, protegiéndose de la luz. La muestra se colocó en una centrífuga (Hermle Labortechnik) a $3000 \mathrm{rpm}$ por $10 \mathrm{~min}$ a $10^{\circ} \mathrm{C}$. La fase orgánica se separó a un tubo falcon. Se incorporaron $5 \mathrm{~mL}$ de agua destilada y se homogenizó en un vortex. El proceso de extracción se repitió bajo las mismas condiciones en el pellet del mesocarpio. La fase orgánica se transfirió a otro falcon y se aforó a $7.5 \mathrm{~mL}$ con éter de petróleo. El extracto se midió a $450 \mathrm{~nm}$ en un espectrofotómetro (Génesis 20-Thermo Spectronic). Para la cuantificación de CT se empleó la ecuación 6.

$$
C=\frac{A_{450} \mathrm{~nm} \times \text { volumen final }(\mathrm{ml}) \times 10^{4}}{2592 \times \text { peso muestra }(\mathrm{g})}
$$

Donde:

C: concentración de carotenos totales $(\mu \mathrm{g} / \mathrm{g})$

$\mathrm{A}_{450 \text { : }}$ absorbancia a $450 \mathrm{~nm}$

104: constante de conservación de unidades $(\mu \mathrm{g} / \mathrm{g})$

2592: coeficiente de extinción molar del $\beta$-caroteno en éter de petróleo.

Ácido L-ascórbico: Se preparó un tampón de fosfato de sodio $100 \mathrm{mM} \mathrm{pH} \mathrm{3.5.} \mathrm{Se} \mathrm{mezclaron} 2 \mathrm{~g}$ de endocarpio triturado congelado y $10 \mathrm{~mL}$ de tampón, durante $10 \mathrm{~min}$, protegiéndose de la luz. La muestra se colocó en una centrífuga (Hermle Labortechnik) a 6000 rpm por $10 \mathrm{~min}$. El sobrenadante se filtró con papel filtro Whatman No.1, y se almacenó a $-20^{\circ} \mathrm{C}$ hasta su análisis. La determinación y cuantificación de AA se realizó según el procedimiento de Megazyme (2005). Por cada muestra a analizar se colocaron en tubos de ensayo distintos 1500 y $1520 \mu \mathrm{L}$ de agua destilada $\left(\sim 37^{\circ} \mathrm{C}\right)$, respectivamente. En cada tubo se añadieron $100 \mu \mathrm{L}$ de muestra y $500 \mu \mathrm{L}$ de tampón de fosfato de sodio $(0.2 \mathrm{M}$ )/citrato de sodio (0.2 M) (muestra). Y sólo en uno de los tubos se agregaron $20 \mu \mathrm{L}$ de la suspensión de ácido ascórbico oxidasa (muestra sin tratar). Independientemente los tubos se mezclaron e incubaron durante $3 \mathrm{~min}$ a $37^{\circ} \mathrm{C}$, homogenizándose cada minuto durante 5 segundos. Se añadieron a cada tubo $200 \mu \mathrm{L}$ de tampón MTT/acetato, se mezclaron e incubaron bajo las mismas condiciones, y se leyeron las absorbancias de la "muestra" y "muestra sin tratar" (A1) a 578 nm en un espectrofotómetro (Génesis 20-Thermo Spectronic). Luego se agregaron $0.20 \mathrm{~mL}$ de la solución PMS, se mezcló y se leyeron las absorbancias de las soluciones $\left(A_{2}\right)$. Se calculó las diferencias de absorción $\left(A_{2}-A_{1}\right)$ con "muestras" y "muestras sin tratar". Se restó la diferencia de absorción de la muestra sin tratar de la

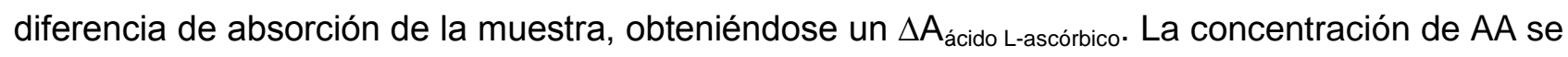
obtuvo por medio de la ecuación 7. 


$$
C=\frac{V \times M W}{\varepsilon \times d \times v} \times \Delta A_{\text {ácido L-ascórbico }}
$$

Donde:

$\mathrm{V}$ : volumen final $[\mathrm{mL}]$

MW: peso molecular del ácido D-ascórbico [g/mol]

$\varepsilon$ : coeficiente de extinción de MTT-formazan a $578 \mathrm{~nm}: 16900\left[\mathrm{l} \mathrm{x} \mathrm{mol}^{-1} \mathrm{x} \mathrm{cm}^{-1}\right]$

$\mathrm{d}$ : paso de luz [cm]

v: volumen de muestra $[\mathrm{mL}]$.

La concentración de AA [g/100g] a partir de la cantidad pesada se obtuvo empleando la ecuación 8.

$$
\text { Contenido de } A A=\frac{\text { Cácido } L \text {-ascórbico }[g / \text { Lsolución de muestra }]}{\text { peso }_{\text {muestra }}[g / \text { Lsolución de muestra }]} \times 100
$$

Capacidad antioxidante total: Los extractos preparados para la determinación de FT se emplearon para comprobar la $C A$, basándose en la decoloración del radical $A B T S^{*+}$, según Re et al. (1999). El radical se formó con $5 \mathrm{~mL}$ de ABTS $7 \mathrm{mM}$ y peroxosulfato de potasio $2.45 \mathrm{mM}$ en agua destilada, dejándose en oscuridad a temperatura ambiente por $16 \mathrm{~h}$. El radical se diluyó con etanol hasta obtener una absorbancia de $0.700 \pm 0.005$, medida a $734 \mathrm{~nm}$. A $1000 \mu \mathrm{L}$ de la dilución del radical se añadieron $20 \mu \mathrm{L}$ de extracto. La mezcla se homogenizó en un vortex y reposó 6 min previo a la lectura de su absorbancia a $734 \mathrm{~nm}$ en un espectrofotómetro (Génesis 20-Thermo Spectronic). La medida es válida al obtener entre un 20 y $80 \%$ de inhibición, comparada con la absorbancia del "blanco", el mismo que se preparó sustituyendo el extracto por etanol. Cada extracto se analizó por triplicado. Para la cuantificación de la CA se realizó una curva de calibración con Trolox $0.5 \mathrm{mM}$.

Análisis estadístico: Se empleó un diseño experimental AxB. Las variables independientes fueron: el estado de maduración y la altura de cultivo. $Y$ las dependientes: los parámetros fisicoquímicos (color superficial e interno, pH, ATT y SST) y bioquímicos (FT, AT, CT, AA y CA). Los resultados se procesaron mediante un análisis de varianza y las medias se compararon con la prueba de Tukey con una significancia de 0.05 usando el software STATGRAPHICS Centurion XV.II.

\section{Resultados y discusión}

3.1. Efecto de la altura de cultivo y del grado de madurez sobre los parámetros de color del fruto

Los cambios en los parámetros de color de tomate de árbol Morado en tres grados de madurez de consumo, pertenecientes a Pelileo (2660 m.s.n.m.) y Chiquicha (2440 m.s.n.m.) se ven 
influenciados por procesos fisiológicos de la maduración del fruto, donde aumenta el color del epicarpio (Prohens et al., 1996), como se presenta en la figura 2. También podría relacionarse con la degradación de la pared celular de los tejidos de la pulpa de frutos maduros (Márquez, Otero, \& Cortés, 2007). Con la maduración hubo una pérdida de $L^{*}$ en el epicarpio de frutos tanto de Pelileo como de Chiquicha. En el mesocarpio esta disminución únicamente ocurrió en frutos de Pelileo, mientras que en el endocarpio en aquellos de Chiquicha. Por lo contrario, existió un aumento de los valores de $\mathrm{C}^{*}$ del epicarpio y mesocarpio de frutos de Chiquicha; en el endocarpio este aumento se registró en frutos de las dos localidades estudiadas. Mientras que, los valores del ángulo de tono del epicarpio y mesocarpio disminuyeron con la maduración, lo que corresponde al cambio de tonalidad de anaranjado-rojizo hacia un rojo-púrpura, y el paso de un tono anaranjado hacia uno más intenso, respectivamente. En el endocarpio los valores en ambas localidades se mostraron más constantes.

Luminosidad $\left(L^{*}\right)$ : El epicarpio de frutos (tres grados de madurez) cultivados en Chiquicha presentó valores superiores de $L^{*}$ que los de Pelileo, pero sólo se encontró diferencia significativa en frutos maduros y sobremaduros de las dos zonas, como se muestra en la tabla 1 . El mesocarpio de tomates de Pelileo obtuvieron valores de $L^{*}$ estadísticamente mayores que los de Chiquicha. En el endocarpio se encontraron diferencias significativas entre las dos zonas sólo en tomates inmaduros, por lo que podría decirse que no habría influencia de la atura de cultivo sobre este parámetro de color. En el epicarpio existió una reducción de $L^{*}$ de frutos inmaduros a sobremaduros del $26 \%$ para Pelileo y del $22 \%$ para Chiquicha. Resultados similares se reportaron en café (Carvajal, Aristizábal, Oliveros, \& Mejía, 2011). Durante la maduración, el mesocarpio de frutos de Pelileo presentó una reducción de L*. García, González, Fernández, \& Lobo (2007) reportaron esta tendencia en mango; mientras que en Chiquicha este parámetro permaneció constante. El endocarpio de frutos de Pelileo tuvo un ligero incremento de $L^{*}$ durante la maduración; lo contrario se encontró en frutos de Chiquicha.

Croma $\left(\mathbf{C}^{\star}\right)$ : En la tabla 1 se puede identificar que el epicarpio, mesocarpio y endocarpio de frutos inmaduros y maduros de Pelileo presentaron mayores valores de $C^{*}$ que los de Chiquicha. Esta diferencia podría deberse al efecto de la altura de cultivo y a la interacción de factores como irradiación alta, clima extremo e incremento de concentraciones de ozono y oxidantes atmosféricos. El valor de $\mathrm{C}^{*}$ se mantuvo estable en el epicarpio y endocarpio de frutos inmaduros y maduros de Chiquicha, pero éste se incrementó en el estado sobremaduro. En los frutos de Pelileo hubo una disminución del valor de $\mathrm{C}^{*}$ en el epicarpio, un incremento en el endocarpio, y se mantuvo constante el valor en el mesocarpio. Al igual que en el epicarpio de frutos de Pelileo, existió una reducción en los valores de $C^{*}$ durante la maduración de frambuesa (Peña, Salinas, \& Ríos, 2006).

Ángulo de tono $\left(\mathbf{h}^{\star}\right)$ : El epicarpio, mesocarpio y endocarpio de los frutos inmaduros de Pelileo presentaron menores valores de $\mathrm{h}^{*}$ que los de Chiquicha. La tabla 1 muestra que el epicarpio de 
frutos inmaduros presentaron mayores valores de $\mathrm{h}^{*}$ que los maduros y sobremaduros tanto de Pelileo como de Chiquicha. El mesocarpio tuvo una ligera disminución de los valores de $\mathrm{h}^{*}$ en las dos zonas de cultivo, lo contrario ocurrió con el endocarpio.

\begin{tabular}{|c|c|c|c|}
\hline $\begin{array}{c}\text { GRADO DE MADUREZ } \\
\text { LOCALIDAD }\end{array}$ & Inmaduro & Maduro & Sobremaduro \\
\hline $\begin{array}{c}\text { Pelileo } \\
\text { (2660 m.s.n.m.) }\end{array}$ & & & \\
\hline $\begin{array}{c}\text { Chiquicha } \\
\text { (2440 m.s.n.m.) }\end{array}$ & & & \\
\hline
\end{tabular}

Figura 2. Tomates de árbol Morados a distintos grados de madurez de consumo

\subsection{Efecto de la altura de cultivo y del grado de madurez sobre las características químicas}

pH: Los frutos inmaduros y maduros cultivados en Chiquicha presentaron mayores valores de $\mathrm{pH}$ que los de Pelileo. En la tabla 2 se observa que el valor de $\mathrm{pH}$ del tomate de árbol de las dos localidades se incrementó con la maduración. Resultados similares se han reportado en tomate de árbol por Márquez et al. (2007) y Mwithiga, Inzoberi, Shitada, \& Nyota (2007). Los valores obtenidos se asemejan al encontrado por Ávila \& Ruales (2007) en el genotipo Morado gigante.

Acidez total titulable: Durante la maduración de los frutos, la ATT disminuyó un 12\% para Pelileo y $9 \%$ para Chiquicha. Resultados similares han sido encontrados en maracuyá, guayaba (Rodríguez, López, \& García, 2010), arándanos (Rodarte, Eichholz, Rohn, Kroh, \& Huyskens, 2008) y tomate de árbol (Contreras, Gamba, \& Fischer, 2007; Márquez et al., 2007). Esta variación se debe a la transformación de ácidos en azúcares durante la maduración (Gil, 2010). No se obtuvieron diferencias significativas en los valores de ATT en frutos sobremaduros.

Sólidos solubles totales: No se encontraron diferencias significativas en las localidades analizadas, excepto en los frutos sobremaduros de Pelileo que presentaron menores SST que los de Chiquicha. Durante la maduración los SST de los frutos aumentaron en un 13\% para Pelileo y $19 \%$ para Chiquicha, como se muestra en la tabla 2. Este comportamiento ha sido comprobado en mora, maracuyá, guayaba, papayuela (Rodríguez et al., 2010) y tomate de árbol (Márquez et al., 2007). 

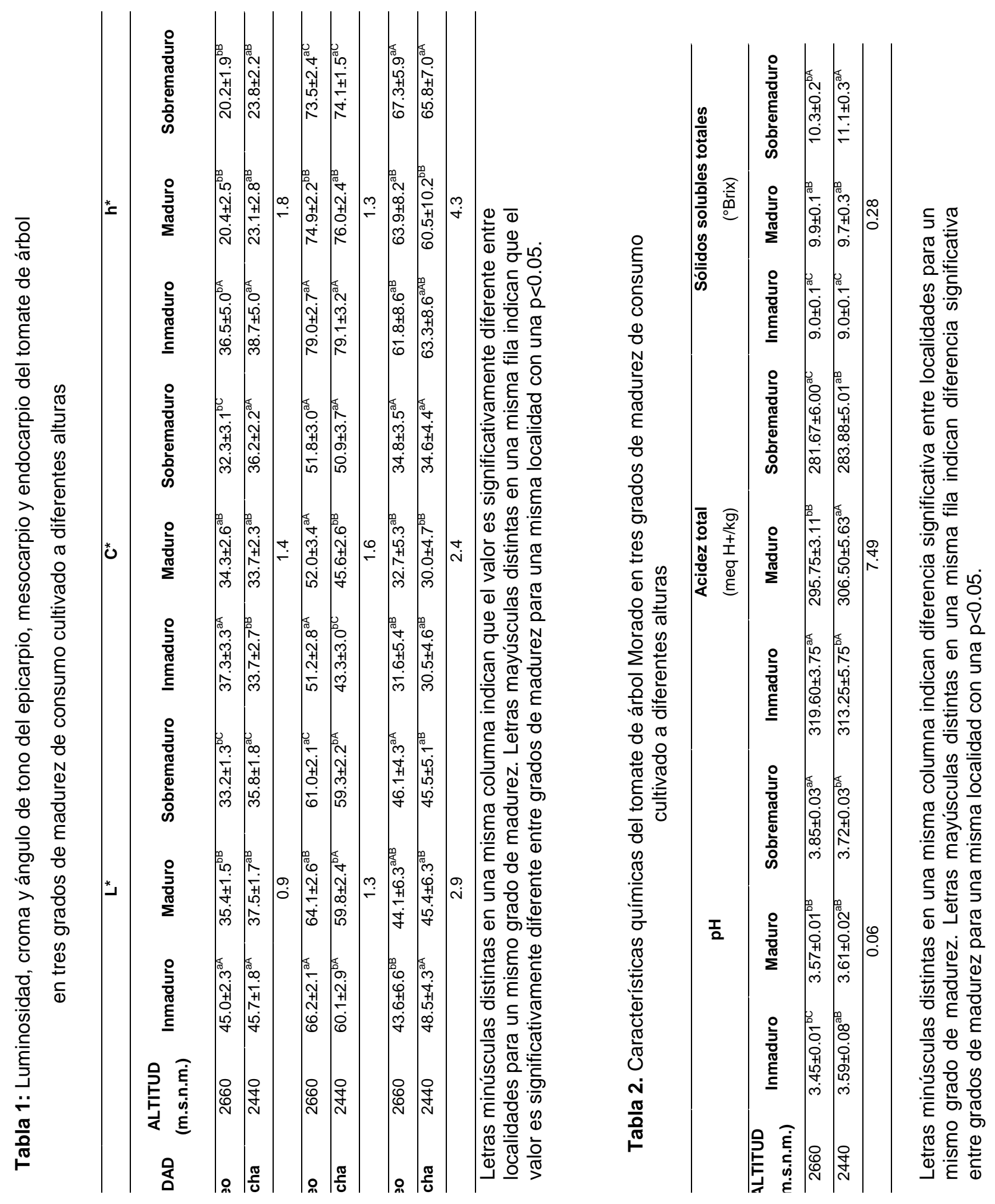
3.3. Efecto de la altura de cultivo y del grado de madurez sobre el contenido de compuestos antioxidantes y la capacidad antioxidante total

Fenoles totales: El endocarpio presentó una concentración de FT tres veces más alta que el mesocarpio, como muestra la figura 3. Existió diferencia significativa entre las zonas estudiadas (efecto de la altura de cultivo) en los estados maduro y sobremaduro del mesocarpio de los frutos. Los frutos de Chiquicha (2440 m.s.n.m.) obtuvieron mayor cantidad de FT. Podría decirse que la altura de Pelileo (2660 m.s.n.m.) afectaría negativamente el contenido de FT. Un efecto similar se reportó en aceite de oliva (Ben et al., 2012; Osman, Metzidakis, Girasopoulos, \& Kiritsakis, 1994). En el endocarpio, sólo en el estado sobremaduro se encontró mayor cantidad de FT en Pelileo. La maduración provocó un aumento del contenido de FT en el mesocarpio, mostrando diferencias significativas entre estados, a excepción de los estados inmaduro y maduro de tomates de árbol de Pelileo. Los valores se incrementaron en un $9 \%$ para Pelileo y $22 \%$ para Chiquicha. En el endocarpio se encontraron diferencias estadísticamente significativas en los tres estados de maduración de las dos zonas. El incremento fue del 21\% para Pelileo y $17 \%$ para Chiquicha. La tendencia de aumento de FT con la madurez, ha sido reportada en bayas de Vaccinium L. (Yuan et al., 2011), uvas moscatel (Lee \& Talcott, 2004), mora, maracuyá, papayuela y guayaba (Rodríguez et al., 2010).

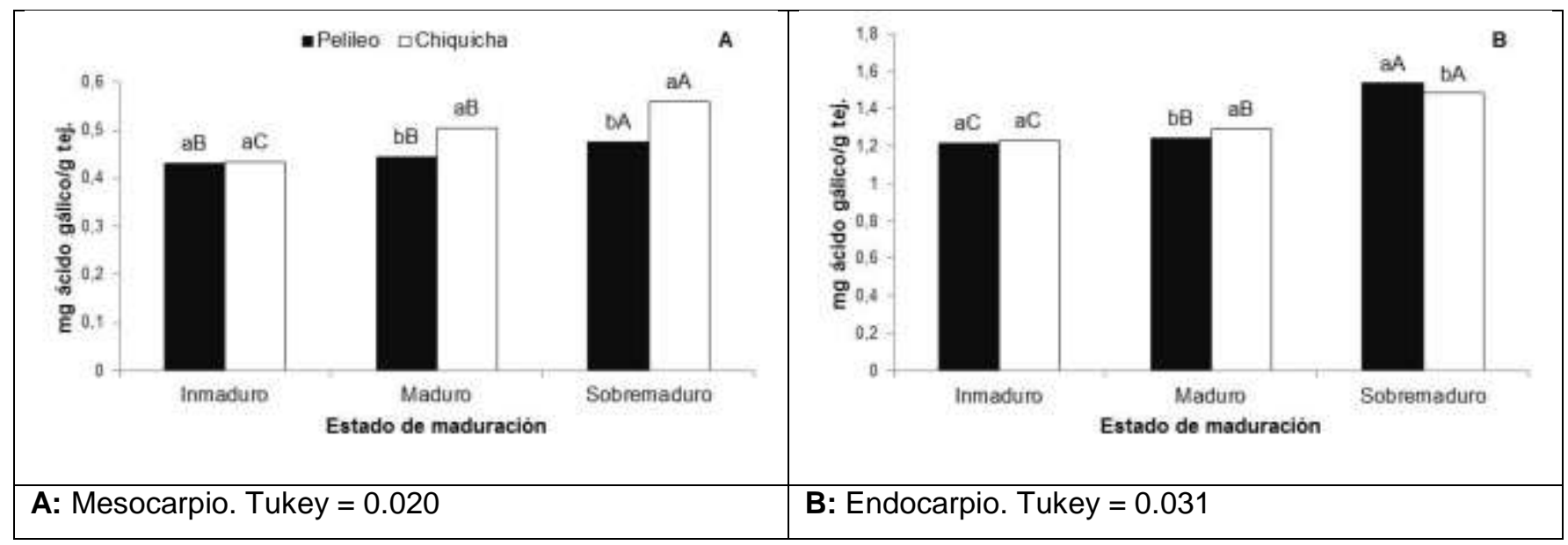

Figura 3: Fenoles totales de tomate de árbol en tres grados de madurez de consumo cultivado a diferentes alturas. Los resultados se expresan como mg equivalentes de ácido gálico/g tej.

Letras minúsculas distintas indican diferencia significativa entre localidades para un mismo grado de madurez. Letras mayúsculas distintas indican diferencia significativa entre grados de madurez para una misma localidad con una $p<0.05$.

Antocianinas totales: Mediante un barrido espectral, como se indica en la figura 4, se evidenció la presencia de AT en el endocarpio del tomate de árbol Morado, cuyos picos más altos se obtuvieron a $530 \mathrm{~nm}$; mientras que una cantidad similar de mesocarpio estudiado no alcanzó absorbancias representativas, determinando así la carencia del pigmento en esta parte del fruto. 


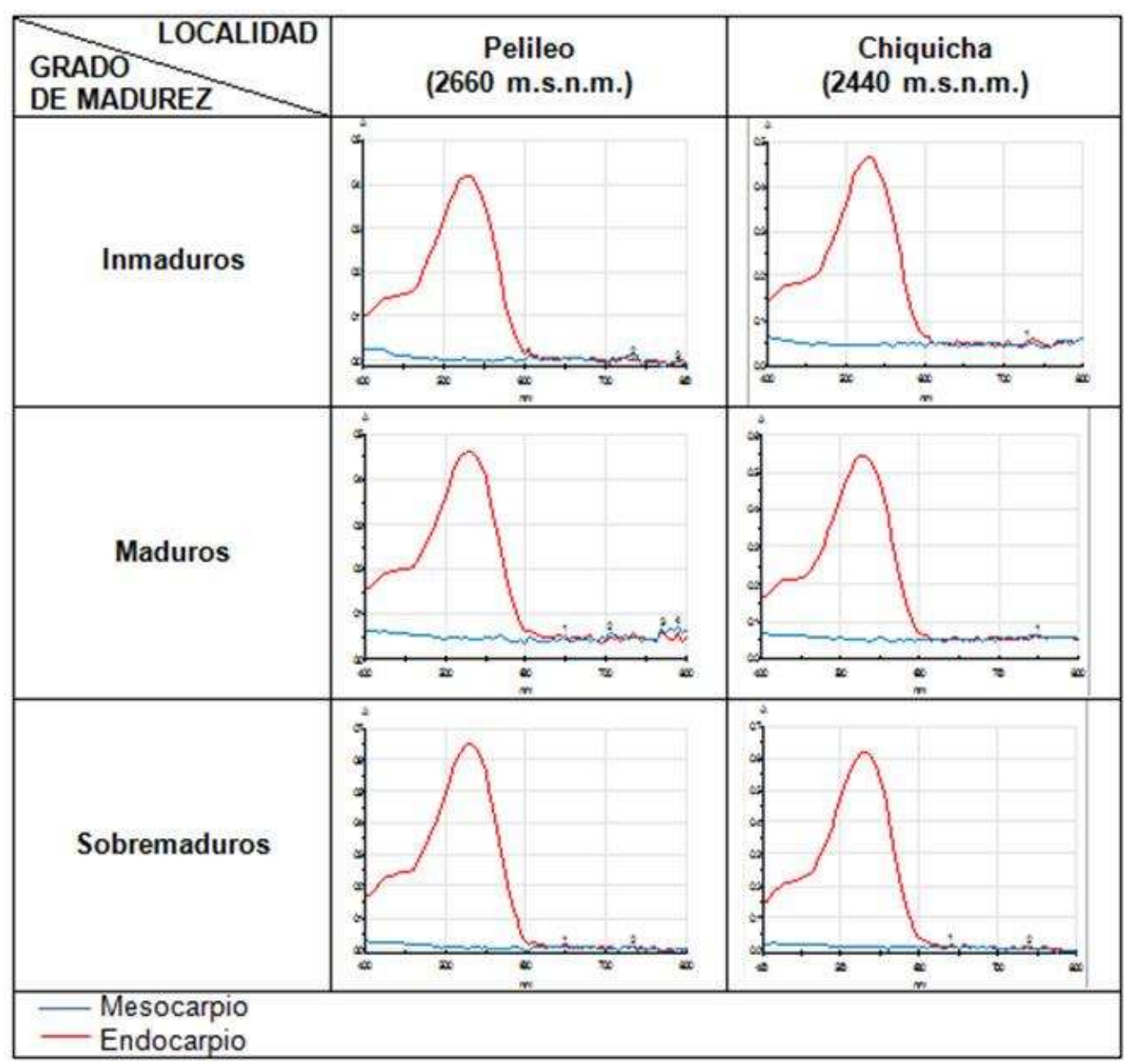

Figura 4. Barrido espectral para la determinación de antocianinas totales en endocarpio y mesocarpio de tomate de árbol en tres grados de madurez de consumo cultivado a diferentes alturas

La Figura 5 indica la influencia de la altura de cultivo en los estados maduro y sobremaduro sobre el contenido de AT. Son escasas las investigaciones que demuestren esta relación; más bien se han enfocado en caracterizar este compuesto. Vera de Rosso \& Mercadante (2007) y Vasco, Ávila, \& Ruales (2009) compararon este pigmento con la delfinidina-3-rutinósido, principal antocianina encontrada en tomate de árbol (Vera de Rosso \& Mercadante, 2007; Wrolstad \& Heatherbell, 1974), representando el $62 \%$ del total del contenido de antocianinas. Entre los estados inmaduro y sobremaduro se halló un incremento del contenido de AT del 33\% para Pelileo y $30 \%$ para Chiquicha. El efecto de la maduración sobre las AT ha sido demostrado en frambuesas (Peña et al., 2006), arándanos (Kalt et al., 2003) y moras Marion y Evergreen (Siriwoharn, Wrolstad, Finn, \& Pereira, 2004). 


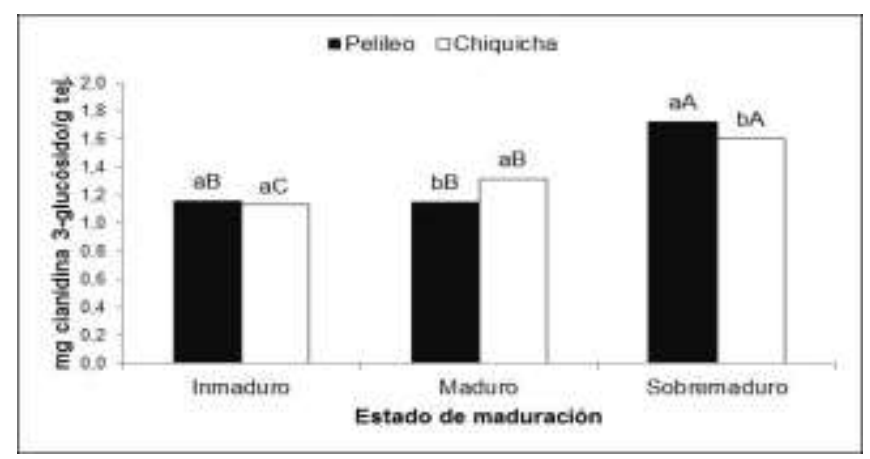

Figura 5: Antocianinas totales del endocarpio de tomate de árbol en tres grados de madurez de consumo cultivado a diferentes alturas. Los resultados se expresan como mg equivalentes de cianidina 3-glucósido/g tej.

Letras minúsculas distintas indican diferencia significativa entre localidades para un mismo grado de madurez. Letras mayúsculas distintas indican diferencia significativa entre grados de madurez para una misma localidad con una $p<0.05$. Tukey $=0.071$

Carotenos totales: El endocarpio presentó una concentración de CT de dos a tres veces más alta que el mesocarpio, como se muestra en la figura 6. Los frutos de Chiquicha tuvieron un porcentaje significativamente mayor (aproximadamente 29\%) de CT en el mesocarpio que los de Pelileo. Un efecto similar se ha reportado en uvilla (Fischer et al., 2000). Los resultados obtenidos en el mesocarpio se asemejan al encontrado por Lister, Morrison, Kerkhofs, \& Wright (2005) en tomate de árbol variedad Roja. En el endocarpio se evidenció la influencia de la altura de cultivo sobre los CT en frutos maduros y sobremaduros, siendo Pelileo la zona que presentó mayor concentración. El efecto de la maduración sobre los CT del endocarpio, provocó diferencia significativa entre estados de maduración y alturas de cultivo estudiadas. Durante la maduración del fruto la concentración de CT aumentó; Repo \& Encina (2008) obtuvieron resultados afines en uvilla. Este incremento en el contenido de CT podría deberse al proceso de carotenogénesis, que puede continuar después de la cosecha siempre que la fruta permanezca intacta (Rodríguez, 1999).

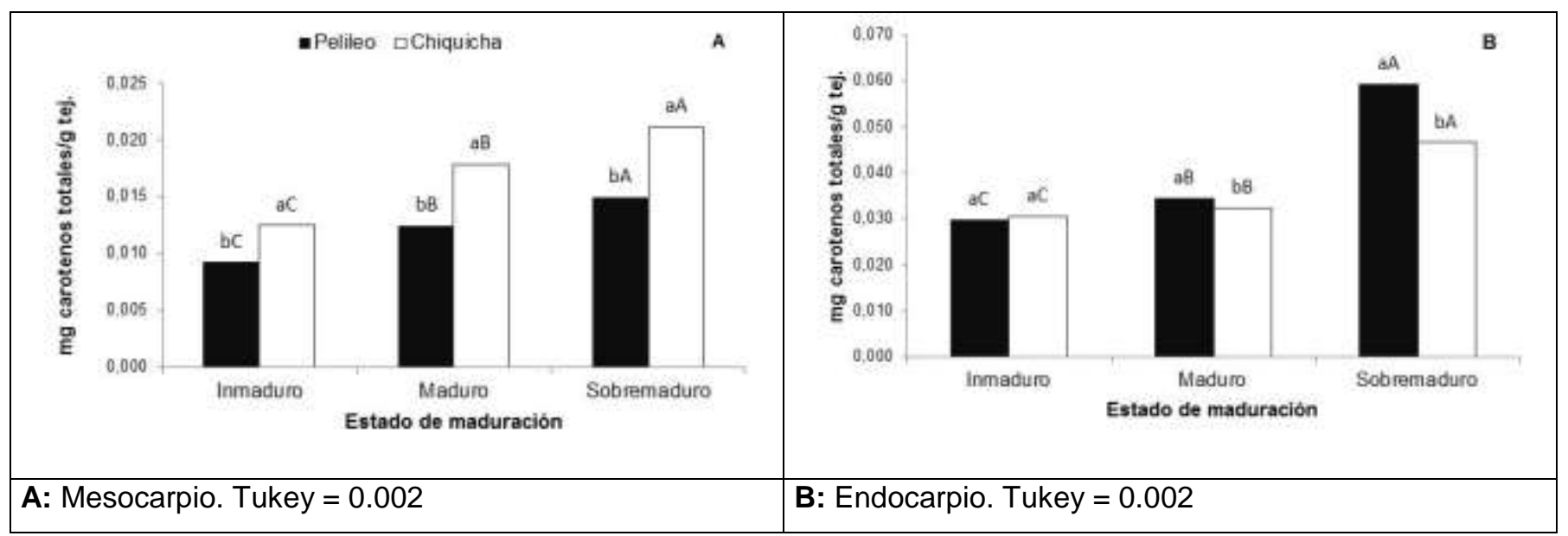

Figura 6: Carotenos totales de tomate de árbol en tres grados de madurez de consumo cultivado a diferentes alturas. Los resultados se expresan como $\mathrm{mg}$ carotenos totales/g tej. 
Letras minúsculas distintas indican diferencia significativa entre localidades para un mismo grado de madurez. Letras mayúsculas distintas indican diferencia significativa entre grados de madurez para una misma localidad con una $p<0.05$.

Ácido L-ascórbico: El contenido de AA fue analizado en el endocarpio de los frutos, pues se conoce que esta zona presenta mayor concentración de ácido ascórbico que el mesocarpio (Gordon, Rodrigues, Marx, \& Papagiannopoulos, 2007). Al analizar el efecto de la altura de cultivo sobre el contenido de AA se encontró diferencia significativa sólo en el estado sobremaduro. Los frutos de Chiquicha presentaron 13\% más AA que los de Pelileo. Probablemente esta diferencia se habría producido por la altura de cultivo entre otros factores, como ha sido reportado en un estudio en papas (Hamouz et al., 2006). Los valores de AA en el endocarpio se encontraron comprendidos entre 0.16 y 0.19 y entre 0.16 y $0.22 \mathrm{mg}$ ácido L-ascórbico/g tej. en frutos de Pelileo y Chiquicha, respectivamente. Vasco et al. (2009) reportaron una concentración de $0.16 \mathrm{mg}$ de ácido ascórbico en tomate de árbol del genotipo Rojo-púrpura. La figura 7 muestra el incremento de AA conforme la maduración avanza. Se han reportado resultados similares en uvilla (Repo \& Encina, 2008) y pimientos (Howard, Talcott, Brenes, \& Villalon, 2000). Lee \& Kader (2000) manifestaron que existen frutos que acumularon AA durante la maduración en la planta o fuera de ella, pero el incremento fue mayor para frutos que se mantuvieron en la planta; e indicaron que hay estudios demostrando que el contenido de AA aumentó con la maduración de ciertos frutos en la planta; y que en general, las frutas recolectadas frescas contienen más vitamina $C$ que las que se mantuvieron en almacenamiento.

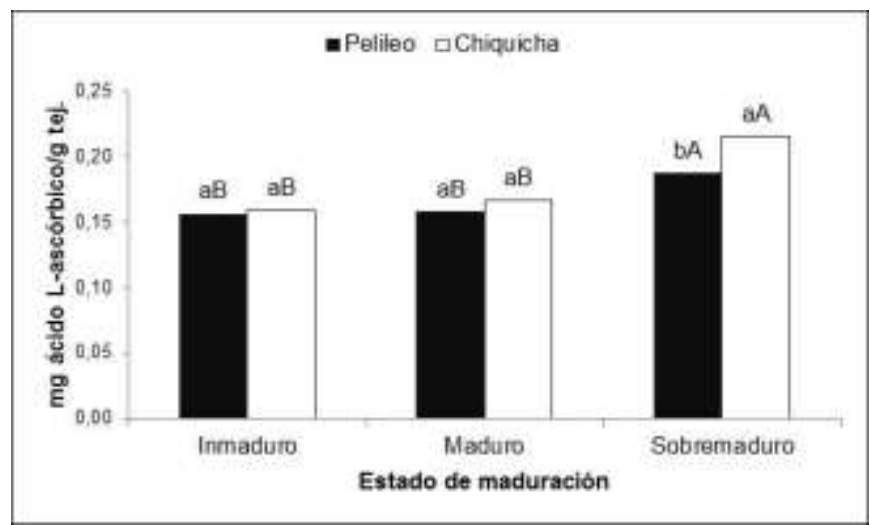

Figura 7: Ácido L-ascórbico del endocarpio de tomate de árbol en tres grados de madurez de consumo cultivado a diferentes alturas. Los resultados se expresan como $\mathrm{mg}$ ácido L-ascórbico/g tej.

Letras minúsculas distintas indican diferencia significativa entre localidades para un mismo grado de madurez. Letras mayúsculas distintas indican diferencia significativa entre grados de madurez para una misma localidad con una $p<0.05$. Tukey $=0.020$.

Capacidad antioxidante total: Existió mayor CA en el endocarpio que en el mesocarpio del tomate de árbol, como se presenta en la figura 8, lo que podría deberse a la presencia de antocianinas, compuestos con conocida capacidad antioxidante (Vasco et al., 2009). En los tres estados de maduración, el mesocarpio de frutos de Pelileo tuvo menos CA que los de Chiquicha, 
lo contrario se obtuvo en el endocarpio. Durante la maduración se encontró un incremento estadísticamente significativo de la CA en el mesocarpio, correspondiente a un $21 \%$ (Pelileo) y $20 \%$ (Chiquicha). Este efecto también se ha reportado en frambuesa (Peña et al., 2006) y uvilla (Repo \& Encina, 2008).

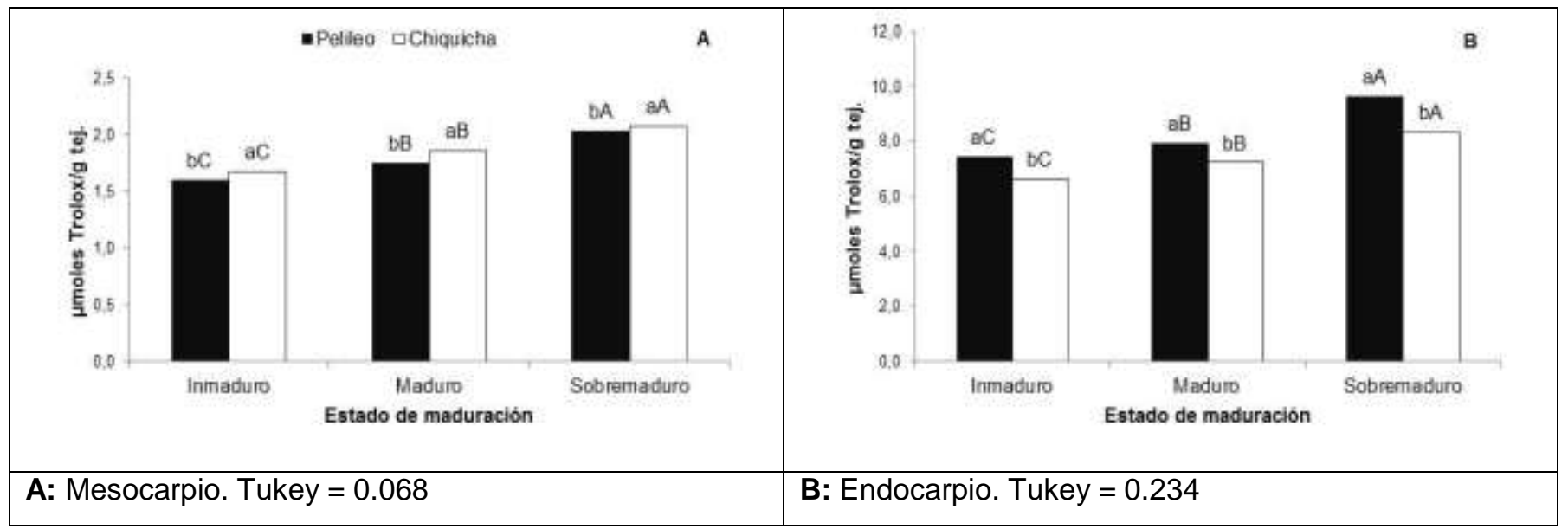

Figura 8: Capacidad antioxidante total de tomate de árbol en tres grados de madurez de consumo cultivado a diferentes alturas. Los resultados se expresan como $\mu$ moles equivalentes de Trolox/g tej.

Letras minúsculas distintas indican diferencia significativa entre localidades para un mismo grado de madurez. Letras mayúsculas distintas indican diferencia significativa entre grados de madurez para una misma localidad con una $p<0.05$.

La CA de un fruto es la suma de las actividades antioxidantes de todos los compuestos presentes en el tejido. En la presente investigación se analizaron los componentes que se encuentran en mayor proporción en el tomate de árbol. Las diferencias encontradas al comparar el mesocarpio y el endocarpio no estarían relacionadas directamente con la altura de las zonas de cultivo y podrían deberse a la presencia de un sinnúmero de familias de compuestos presentes en el fruto y a factores abióticos, que además de la altura de cultivo influyeron sobre sus compuestos bioactivos.

\section{Conclusiones y Recomendaciones}

Los frutos sobremaduros de las dos zonas de cultivo tuvieron mayor concentración de compuestos bioactivos y CA, esto estaría relacionado con la disminución de la humedad (pérdida de peso) que es evidenciada por el arrugamiento del epicarpio. En general, los tomates de árbol de Chiquicha (2440 m.s.n.m.) obtuvieron mayor concentración de compuestos antioxidantes que los de Pelileo (2660 m.s.n.m.). Posiblemente esta respuesta se deba a que dicha plantación, constituida por al menos dos genotipos, lleva mayor tiempo en producción, y se sitúa en una zona montañosa del cantón Pelileo con buenas características para el cultivo de este frutal. Estos factores podrían haber influido en su composición; sin embargo, hacen falta estudios bioquímicos que ayuden a elucidar el efecto de la altura de cultivo sobre el metabolismo del fruto, tomando en cuenta que el material vegetal deberá pertenecer a cultivos que lleven tiempos similares en producción. También 
se recomienda estudiar la influencia de la altitud de la plantación y otros factores abióticos sobre el contenido de compuestos bioactivos y la actividad enzimática antioxidante de los frutos.

\section{Bibliografía}

Arango, J. (2006). Análisis de expresión de los genes de la ruta biosintética de carotenos, y cuantificación de carotenos en hojas y raíces de plantas de yuca a diferentes edades. Pontificia Universidad Javeriana, Bogotá.

Ávila, J., \& Ruales, J. (2007). Caracterización postcosecha de 4 genotipos de tomate de árbol (Solanum betaceum Cav.) cultivados en Ecuador. Alimentos Ciencia e Ingeniería, 16(3), 138-140.

Beas, R., Loarca, G., Guzmán, S., Rodríguez, M., Vasco, N., \& Guevara, F. (2011). Potencial nutracéutico de componentes bioactivos presentes en huitlacoche de la zona centro de México. Revista Mexicana de Ciencias Farmacéuticas, 42(2), 36-44.

Ben, N., Abaza, L., Youssef, O., Naeit, S., Nizard, D., Chedly, A., \& Mokhtar, Z. (2012). Influence of the site of cultivation on Chétoui Olive (Olea europea L.) oil quality. Plant Production Science, 15(3), 228-237.

Carvajal, J., Aristizábal, I., Oliveros, C., \& Mejía, J. (2011). Colorimetría del fruto de café (Coffea arabica L.) durante su desarrollo y maduración. Revista Facultad Nacional de Agronomía, Medellín, 64(2), 6229-6240.

Coleto, J. (1994). Crecimiento y desarrollo de las especies frutales (2a ed.). Madrid: Mundi-Prensa.

Contreras, J., Gamba, H., \& Fischer, G. (2007). Características fisicoquímicas y organolépticas de frutos de tomate de árbol (Solanum betaceum Cav) a dos tipos de almacenamiento y tipos de cera. Ciencia y Agricultura, 5(2), 39-49.

Chalampuente, D., \& Prado, P. (2005). Caracterización morfoagronómica y molecular de la colección de tomate de árbol (Cyphomandra betacea Sendt) del banco de germoplasma del INIAP, Ecuador. Pontificia Universidad Católica del Ecuador Sede Ibarra, Ibarra.

Fischer, G., Ebert, G., \& Lüdders. (2000). Provitamin A carotenoids, organic acids and ascorbic acid content of cape goosseberry (Physalis peruviana L.) ecotypes grown at two tropical altitudes Artículo presentado en ISHS Acta Horticulturae 531: II ISHS Conference on fruit production in the tropics and subtropics.

García, González, M., Fernández, D., \& Lobo, M. (2007). Comportamiento postcosecha de dos cultivares tardíos de mango. Artículo presentado en $\mathrm{V}$ Congreso lberoamericano de Tecnología Postcosecha y Agroexportaciones, Universidad Politécnica de Cartagena. 
Gil, Á. (2010). Tratado de nutrición. Tomo II. Composición y calidad nutritiva de los alimentos (2a ed.). Madrid: Panamericana.

Gordon, A., Rodrigues, R., Marx, F., \& Papagiannopoulos, M. (2007). Antioxidant capacity of tamarillo fruit (Cyphomandra betacea). Witzenhausen: University of Bonn, Institute of Nutrition and Food Sciences.

Hamouz, K., Lachman, J., Dvorak, P., Cepl, J., \& Sarec, P. (2006). Influence of site conditions and cultivars on the contents of antioxidants in potato tubers. Zeszyty Problemowe Postepow Nauk Rolniczych, 511(1), 245-254.

Hecke, K., Tausz, M., Gigele, T., Havranek, W. M., Anfodillo, T., \& Grill, D. (2003). Foliar antioxidants and protective pigments in Larix decidua Mill. from contrasting elevations in the northern and southern Tyrolean Limestone Alps. European Journal of Forest Research, 122(6), 368-375.

Howard, L., Talcott, S., Brenes, C., \& Villalon, B. (2000). Changes in phytochemical and antioxidant activity of selected pepper cultivars (Capsicum Species) as influenced by maturity. Journal of Agricultural and Food Chemistry, 48(5), 1713-1720.

Kalt, W., Lawand, C., Ryan, D., McDonald, J., Donner, H., \& Forney, C. (2003). Oxygen radical absorbing capacity, anthocyanin and phenolic content of highbush blueberries (Vaccinium corymbosum L.) during ripening and storage. Journal of the American Society of Horticultural Science, 128(6), 917-923.

Lee, \& Kader, A. (2000). Preharvest and postharvest factors influencing vitamin C content of horticultural crops. Postharvest Biology and Technology, 20, 207-220.

Lee, \& Talcott, S. (2004). Fruit maturity and juice extraction influences ellagic acid derivatives and other antioxidant polyphenolics in muscadine grapes. Journal of agricultural and food chemistry, 52(2), 361-366.

Lister, C., Morrison, S., Kerkhofs, N., \& Wright, K. (2005). The nutricional composition and health benefits of New Zealand tamarillos. Christchurch.

Márquez, C., Otero, C., \& Cortés, M. (2007). Cambios fisiológicos, textuales, fisicoquímicos y microestructurales del tomate de árbol (Cyphomandra betacea S.) en poscosecha. Vitae, Revista de la Facultad de Química Farmacéutica, 14(2), 9-16.

Megazyme. (2005). Ácido L-Ascórbico (L-Ascorbato). Procedimiento de ensayo. In M. I. I. Ltd. (Ed.). Irlanda. 
Mwithiga, G., Inzoberi, M., Shitada, D., \& Nyota, P. (2007). Evaluation of the effect of ripening on the sensory quality and propierties of tamarillo (Cyphomandra betaceae) fruits. Journal of Food Engineering, 79, 117-123.

Osman, M., Metzidakis, I., Girasopoulos, G., \& Kiritsakis, A. (1994). Quantitative changes in olive oil fruits collected from trees grown at two altitudes. Riv. Ital. Sostanze Grasse, 71, 187189.

Paltrinieri, G., Figuerola, F., \& Rojas, L. (1993). Procesamiento de frutas y hortalizas mediante métodos artesanales y de pequeña escala. Santiago.

Peña, G., Salinas, Y., \& Ríos, R. (2006). Contenido de antocianinas totales y actividad antioxidante en frutos de frambuesa (Rubus idaeus L.) con diferente grado de maduración. Revista Chapingo. Serie horticultura, 12(2), 159-163.

Prohens, J., Ruiz, J., \& Nuez, F. (1996). Advancing the tamarillo harvest by induced postharvest ripening. American Society for Horticultural Science, 31(1), 109-111.

Ramírez, T. (2009). Perfil de tomate de árbol. In CORPEI (Ed.).

Re, R., Pellegrini, N., Proteggente, A., Pannala, A., Tang, M., \& Rice-Evans, C. (1999). Antioxidant activity applying an improved ABTS radical cation decolorization assay. Free radical biology \& medicine, 26(9/10), 1231-1237.

Repo, R., \& Encina, R. (2008). Determinación de la capacidad antioxidante y compuestos bioactivos de frutas nativas peruanas. Revista de la Sociedad Química del Perú, 74(2), 108-124.

Revelo, Pérez, E., \& Maila, M. (2004). El cultivo de tomate de árbol. Quito.

Reyes, R., \& Sanabria, O. (1993). Tomate de árbol. Cyphomandra betacea (Cav) Sendtn. Recuperado de http://www.ibiologia.unam.mx/jardin/gela/page13.html

Rodarte, A., Eichholz, I., Rohn, S., Kroh, L., \& Huyskens, S. (2008). Phenolic profile and antioxidant activity of highbush blueberry (Vaccinium corymbosum L.) during fruit maturation and ripening. Food Chemistry, 109, 564-572.

Rodríguez. (1999). Carotenoides y preparación de alimentos: La retención de los carotenoides provitamina $A$ en alimentos preparados, procesados y almacenados. Campinas.

Rodríguez, López, L., \& García, M. (2010). Determinación de la composición química y actividad antioxidante en distintos estados de madurez de frutas de consumo habitual en Colombia, mora (Rubus glaucus B.), maracuyá (Passiflora edulis S.), guayaba (Psidium guajava L.) y papayuela (Carica cundinamarcensis J.). Revista Alimentos Hoy, Colombia, 19(21). 
Sakata, T., Nakato, T., \& Yokoi, Y. (2006). Altitudinal changes in Rubisco and APX activities in Aconogonum weyrichii in the alpine region of Mt. Fuji. Polar Bioscience Journal, 19, 115122.

Sanz, J., \& Gallego, R. (2001). Diccionario akal del color. Madrid.

Singleton, V., \& Rossi, J. (1965). Colorimetry of total phenolics with phosphomolybdicphosphotungstic acid reagents. Amer. J. Enol. Viticult., 16, 144-158.

Siriwoharn, T., Wrolstad, R., Finn, C., \& Pereira, C. (2004). Influence of cultivar, maturity, and sampling on blackberry (Rubus L. Hybrids) anthocyanins, polyphenolics, and antioxidant properties. Journal of Agricultural and Food Chemistry, 52(26), 8021-8030.

Vasco, C., Ávila, J., \& Ruales, J. (2009). Physical and chemical characteristics of golden-yellow and purple-red varieties of tamarillo fruit (Solanum betaceum Cav.). International Journal of Food Sciences and Nutrition.

Vera de Rosso, V., \& Mercadante, A. (2007). HPLC-PDA-MS/MS of anthocyanins and carotenoids from dovyalis and tamarillo fruits. Journal of Agricultural and Food Chemistry, 55(22), 91359141.

Wrolstad, R., \& Heatherbell, D. (1974). Identification of anthocyanins and distribution of flavonoids in tamarillo fruit (Cyphomandra betaceae (Cav.) Sendt.). Journal of the Science of Food and Agriculture, 25, 1221-1228.

Yuan, W., Zhou, L., Deng, G., Wang, P., Creech, D., \& Li, S. (2011). Anthocyanins, phenolics, and antioxidant capacity of Vaccinium L. in Texas, USA. Pharmaceutical Crops, 2, 11-23. 